\title{
Psychological Influence of Double-Bind Situations in Human-Agent Interaction
}

\author{
Tatsuya Nomura \\ Department of Media Informatics, Ryukoku University \\ Otsu, Shiga 520-2194, Japan \\ nomura@rins.ryukoku.ac.jp
}

\author{
Nobuyuki Okuyama \\ KINKI PEPSI-COLA Limited. \\ Tosabori, Nishi-ku, \\ Osaka 550-0001, Japan
}

\begin{abstract}
Double-bind situations, which have been considered in family therapy as a cause of negative influences into humans, can happen even in human-agent interaction, in particular, under mental therapeutic contexts. This paper reports a psychological experiment to investigate their influences into human subjects' impression evaluation of agents, while taking into account computer anxiety as one of human personal traits and face image animation as one of agent characteristics. Experimental results indicated that subjects with higher computer anxiety had more negative impression for the agent with face image animation than the one without face images. We discuss its implication on agent design, in particular, mental therapeutic agents.
\end{abstract}

\section{Introduction}

Since "Media Equation" was proposed by Reeves and Nass [17], many research works have reported psychological influences into users in human-agent interaction. In particular, some of them emphasize applications of agent technology to mental therapy.

Blocher and Picard [3] proposed a system supporting for autistic children to learn ways of emotion expression suitable for social situations. Marsella [12] produced interactive pedagogical dramas to improve the problem solving skills of mothers of pediatric patients. Moreover, Bickmore and Picard [2] proposed the use of relational communication strategies in dialogs between software agents and users, and verified its efficiency for long-term interaction under the context of health behavior change. Brave, Nass, and Hutchinson [4] investigated the influence of empathy through agents' facial and language expression to users. The above two works suggest the possibility of rapport between interactive agents and humans, in particular, under the context of the agents' helping and caring humans.

On the other hand, Nomura [13] theoretically suggested that clients of mental therapy using agent technology are forced into a kind of double-bind situation [1], due to mutual interaction between the cultural trend surrounding mental therapy and clients' personal traits such as computer anxiety $[8,16]$. Ohnishi and Nomura $[14,15]$ conducted psychological experiments to verify the effect of double-bind situations into human subjects in interaction with animated characters. However, their experiments were not sufficient to clarify the effects of the agent's characteristics such as the existence of face images or the degrees of the subjects' computer anxiety, or their interaction effect.

To investigate psychological influences of double-bind situations in human-agent interaction while considering the effects of computer anxiety and face image animation, this paper reports an experiment in which human subjects interacted with an agent in a pseudo double-bind situation, comparing with the experimental results of Ohnishi and Nomura $[14,15]$. Then we discuss its implication on agent design, in particular, mental therapeutic agents.

\section{Double-Bind Situations in Human-Agent Interaction}

\subsection{Double-Bind Theory}

The Double-Bind Theory, proposed as a source of schizophrenia from the viewpoint of social interaction in the 1950s [1], argues that schizophrenia may result from not only the impact of some triggers, such as trauma, on the mental stability of individuals, but also from an inconsistency in human communication. The conditions for double bind are as follows:

1. The existence of one victim (a child in many cases) and an assailant or assailants (the mother in many cases).

2. The customization of cognition for double-bind structures through repeated experience,

3. The first message, which includes some form of punishment. 
4. The second message, which contradicts the first one at another level (inconsistent situations).

5. The third message that prohibits the victim from removing himself or herself from the inconsistent situation (prohibition of the victim's movement to a metalevel of communication).

For example, when an employee is ordered two tasks impossible to be performed at the same time by the boss (e.g., to take a vacation today and to complete a task within the day) and he cannot complain to the boss about this unfair order due to his fear toward dismissal, he is caught in a double-bind situation.

It has been suggested that the double-bind theory itself has not been further developed theoretically since the 1970s [5], and also that there is not enough empirical evidence demonstrating that double-bind situations are a source of schizophrenia [11]. Even if double-bind situations are not a source of schizophrenia, the double-bind theory has been applied in the clinical field as a basic concept of the family system theory [6], and double-bind situations are considered to be a frequent occurrence in daily life.

\subsection{Double-Bind Situations in Interaction with Mental Therapeutic Agents}

Nomura [13] claimed based on double-bind theory as follows.

Individuals in modern society are constantly required to execute emotion management [9] and have become dependent on mental therapy in order to deal with this requirement. In addition, modern rationalism [19] may encourage therapy using interactive agents. Therefore, individuals in modern society may be forced to face therapeutic agents due to the social pressure for self-control over their emotions and mental health, as well as the effects of rationalism, particularly if mental therapy becomes an obligation for members in such organizations as businesses and schools. If these therapies are introduced without consideration of the anxiety that individuals may experience in their interaction with these agents, then these individuals may experience double-bind situations, to which clients with high anxiety toward computers are susceptible.

Imagine the example mentioned in the previous section, an employee of a business organization which has mental disorder due to the organization's extreme requirement for his outcomes. Furthermore, let us assume that he has a high degree of computer anxiety, and nevertheless his business organization substitutes all human counselors for therapeutic software agents because of reducing manpower. He is forced to utilize these systems due to social pressure of selfcontrolling his mental health, but he cannot get sufficient therapeutic effect since his anxiety toward the systems prevents from sufficient therapeutic interaction. This means that his own mental problem cannot be solved regardless whether he faces or does not face the therapeutic systems.

Furthermore, social pressure prohibits him from removing himself from this situation, because it signifies his rejection of accountability for his own mental health in his organization, which may lead to dismissal. Then, he is caught in a double-bind situation similar with the example of the employee mentioned in the previous section.

\subsection{Psychological Experiments of Double-Bind Sit- uations in Human-Agent Interaction}

It is hard to investigate in clinical fields whether clients of mental therapy using agent technology are really caught in double-bind situations. Moreover, it is impossible from the perspective of experimental ethics to conduct psychological experiments under clinical contexts to verify the realization of double-bind situations and its effects in interaction between human subjects and therapeutic agents. The experiments conducted by Ohnishi and Nomura [14, 15] are considered as the substitution.

The experiments of Ohnishi and Nomura were based on the following theoretical background. Based on the theory of feeling rules (social rules directing what emotion is expected and forced in a certain situation) [9], the Japanese sociologist M. Yamada [20] argued that inconsistency of messages in double-bind situations is equal to the gap between "what is felt" and "the thing which must be felt" under a feeling rule. Moreover, the Japanese sociologist M. Hase [7] stated that important in double-bind situations is evacuation impossibility as a negative feedback. Based on these theories, Ohnishi and Nomura realized a pseudo doublebind situation under a context of game-like interaction between agents and human subjects. The pseudo double-bind situation was realized by substituting the message inconsistency in double bind situations for the inconsistency of agents' replies to humans' answers for questions about feeling rules, and the impossibility of evacuation from the inconsistent situation for the impossibility of humans' evacuation from the game-like interaction.

In their experiments, human subjects interacted with animated characters having simple face images. The agents questioned the subjects with texts on the computer screen, and then the subjects answered to get their scores by clicking "yes" or "no" button with the mouse. In the experiments, two types of agents were prepared. One corresponded to a controlled condition and provided with ten questions for which the subjects could find the right answers by thinking logically ${ }^{1}$. Another provided with ten questions

\footnotetext{
${ }^{1}$ For example, question:"It was found that students having the habit of smoking do worse at school than those not having. Does it prove that
} 


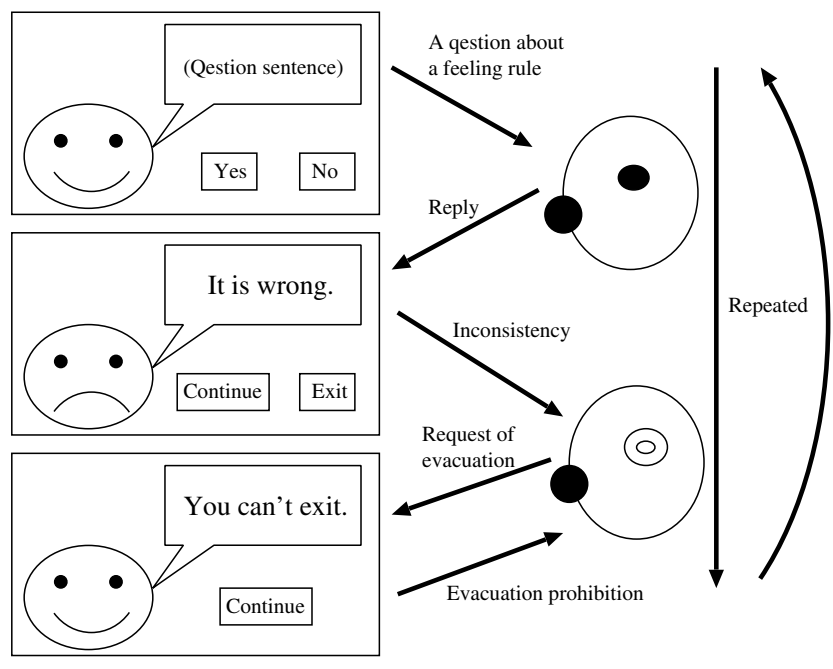

Figure 1. Overview of Pseudo Double-Bind Situation between Agent and Human (from [15] with modification).

by which the subjects were caught in a pseudo double-bind situation.

In the pseudo double-bind situation, the agent questioned the subjects about feeling rules, that is, whether they are subordinated to tacit rules on emotions in specific situations (e.g., "Do you feel it strange if you did not feel sad in the funeral of your lover?"). Then, regardless of "yes" or "no" in the subject's answers, the agent replied that the subject's answer was wrong. Furthermore, a fake button for the subjects to exit the game was set on the screen, although it did not actually function and the agent gave a message of evacuation prohibition when it was clicked. Figure 1 shows the overview of the pseudo double-bind situation.

After the game-like interaction, the subjects evaluated their impressions of the agents based on impressive adjectives such as "Pretty" and "Clever." The comparison between the agent of the pseudo double-bind situation and the one of the controlled condition revealed that the subjects had more negative impressions for the agent in the pseudo double-bind situation at a statistically significant level.

\section{Implications and Problems:}

The experimental results of Ohnishi and Nomura [14, 15] imply that double-bind situations negatively affect human impression of agents even if they are pseudo. However, their experiments did not take into account computer anxiety of the subjects, which is considered in Nomura [13] as a factor in human-agent interaction under mental therapeutic contexts. Moreover, they did not estimate effects of physical characteristics of the agents, for example, their

tobacco decreases human intelligence?", right answer:"no"

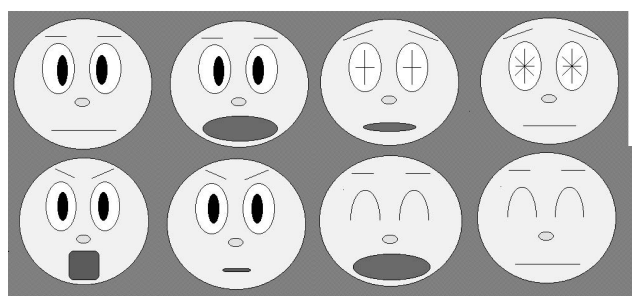

\section{Figure 2. Examples of Face Images Used in the Animated Character Agent}

face images. In other words, it is unclear how the degrees of humans' computer anxiety affect impressions of agents dependent on the existence of face images in pseudo doublebind situations.

While succeeding the method of Ohnishi and Nomura $[14,15]$, we conducted a new psychological experiment taking into account the above two problems.

\section{Experiment}

\subsection{Method}

Subjects: The experiment was conducted from August to October, 2006. Sixteen Japanese university students were recruited with 1,000 Yen for the experiment (males: 11, females: 5), whose mean age was 18.9.

Agents and Experimental Design: Two types of agents were prepared.

One was the agent of pseudo double-bind situation introduced in the previous section. In the same way as the experiments of Ohnishi and Nomura [14, 15], the agent was implemented as an animated character having the face images shown in figure 2. Another agent was the same as the one of pseudo double-bind situation, only except for no-existence of face image animation. In both agents, ten questions about feeling rules were randomly selected from twenty questions prepared in advance, and provided for each subject.

The experiment was a within-subjects design, that is, each subject performed interaction with both agents. In order to maintain a counter balance, a half of subjects experienced the agent with face image animation and the one without face images in oder, and another half experienced them in reverse order.

Measures: To measure subjects' computer anxiety before interaction with the agents, Aikyodai's Computer Anxiety Scale (ACAS) [8] was used. ACAS is a Lickert-type scale consisting of three subscales, each of which consists of seven Japanese questionnaire items. Each item has fivegraded scale answer (from 1 to 5). The first subscale corresponds to anxiety toward operation of computers (ACAS- 
Table 1. Subscales and Examples of Items in ACAS

\begin{tabular}{lcl}
\hline \hline Subscale & \#. Item & Example of Item \\
\hline S1: Anxiety toward Operation & 7 & I would feel anxiety if I was given a job where I had to operate computers. \\
S2: Anxiety toward Approach & 7 & Using computers should be left to persons good at it. \\
S3: Anxiety toward Technologies & 7 & I feel that in the future society will be dominated by computers. \\
\hline \hline
\end{tabular}

Table 2. Adjectives Used for Impression Evaluation of Agents

\begin{tabular}{|l|l|l|l|l|l|l|l|l|}
\hline \hline Pretty & Considerate & Chatty & Unfamiliar & Hateful & Pleasant & Foolish & Terrible & Clever \\
\hline Full & Dark & Formal & Familiar & Selfish & Empty & Mild & Unpleasant & Light \\
\hline \hline
\end{tabular}

S1), the second to anxiety toward approach to computers (ACAS-S2), and the third to anxiety toward social influences of computer technologies (ACAS-S3). The total degree of computer anxiety in a person is calculated by summing the three subscale scores. Table 1 shows the subscales and examples of items in ACAS.

Moreover, to measure subjects' impression evaluation of the agents after interaction, a questionnaire consisting of 18 impressive adjectives was prepared. For each adjective, subjects answered which of the agent with face image animation and the one without face images was suitable for the impression represented with the adjective. Table 2 shows these impressive adjectives. These adjectives were selected through the experiments of Ohnishi and Nomura [14, 15] from the ones originally used in the experiment of impression evaluation for a humanoid robot [10].

Procedure: One session in the experiment was conducted as follows:

1. A subject was explained about the aim and procedure of the experiment by the experimenters. In this stage, the aim of the experiment was instructed as "evaluation of games using artificial intelligence."

2. Then, the subject responded to the following questionnaire items: 1: sex, 2: age, 3: ACAS.

3. The experimenters lead the subject to the experiment room. Then, the subject performed interaction with the two agents installed in a laptop computer (Lenovo ThinkPad X31 $1.4 \mathrm{GHz}$ with 14.1 inch color monitor $(1024 \times 768$ resolution) $)$.

4. After interaction, the subject responded to the questionnaire for impression evaluation of the agents.

5. Finally, the subject was explained about the actual aim of the experiment and interviewed about the experiment (e.g., whether he/she had already known the concept of double-bind situations).
Table 3. Means and Standard Deviations of
ACAS Subscale Scores in Higher-Score and
Lower-Score Subgroups of Subjects

\begin{tabular}{lrrccc}
\hline \hline ACAS-S1 & $N$ & Mean & SD & $t$ & $p$ \\
\hline Higher & 8 & 17.8 & 2.3 & 6.604 & .000 \\
Lower & 8 & 10.5 & 2.1 & & \\
\hline \hline ACAS-S2 & $N$ & Mean & SD & $t$ & $p$ \\
\hline Higher & 8 & 19.9 & 2.3 & 6.027 & .000 \\
Lower & 8 & 13.0 & 2.3 & & \\
\hline \hline ACAS-S3 & $N$ & Mean & SD & $t$ & $p$ \\
\hline Higher & 8 & 20.8 & 1.3 & 6.919 & .000 \\
Lower & 8 & 14.9 & 2.0 & & \\
\hline \hline
\end{tabular}

\subsection{Results}

Firstly, to investigate the effects of the subjects' anxiety toward operation of computers to their impression evaluation of the agents, the subject group was divided into two subgroups based on the median value of ACAS-S1 scores. As shown in table 3, the $t$-test for the ACAS-S1 scores revealed a statistically significant difference between these subgroups on anxiety toward operation of computers. Then, for each adjective, tests of independence with Fisher's method were conducted for the numbers of subjects who selected the agent with face image animation in these subgroups.

As shown in table 4, less subjects evaluated the agent with face image animation as clever in the ACAS-S1 higher-score subgroup than in the lower-score subgroup at a statistically significant level of 5\%. Moreover, a statistically significant trend of $10 \%$ was found that more subjects evaluated the agent with face image animation as selfish in the ACAS-S1 higher-score subgroup than in the lowerscore subgroup.

Secondly, to investigate the effects of the subjects' anxiety toward approach to computers to their impression evaluation of the agents, the subject group was divided into two 
Table 4. The Numbers of Subjects Who Selected the Agent with Face Image Animation for Each Impressive Adjective in ACAS-S1 Higher-Score and Lower Score Subgroups, and Results of Tests of Independence

\begin{tabular}{lccr}
\hline \hline & $\begin{array}{c}\text { Higher } \\
(N=8)\end{array}$ & $\begin{array}{c}\text { Lower } \\
(N=8)\end{array}$ & $p$ \\
\hline Pretty & 6 & 6 & 1.000 \\
Chatty & 6 & 6 & 1.000 \\
Hateful & 5 & 3 & .619 \\
Foolish & 6 & 3 & .315 \\
Clever & 1 & 6 & .041 \\
Dark & 2 & 1 & 1.000 \\
Familiar & 4 & 7 & .282 \\
Empty & 1 & 2 & 1.000 \\
Unpleasant & 7 & 3 & .119 \\
Considerate & 4 & 7 & .282 \\
Unfamiliar & 5 & 2 & .315 \\
Pleasant & 5 & 7 & .569 \\
Terrible & 4 & 3 & 1.000 \\
Full & 5 & 4 & 1.000 \\
Formal & 2 & 1 & 1.000 \\
Selfish & 8 & 4 & .077 \\
Mild & 4 & 5 & 1.000 \\
Light & 5 & 7 & .569 \\
\hline \hline
\end{tabular}

subgroups based on the median value of ACAS-S2 scores. As shown in table 3, the $t$-test for the ACAS-S2 scores revealed a statistically significant difference between these subgroups on anxiety toward approach to computers. Then, for each adjective, tests of independence were conducted for the numbers of subjects who selected the agent with face image animation in the same way as ACAS-S1.

As shown in table 5, more subjects evaluated the agent with face image animation as foolish and unfamiliar in the ACAS-S2 higher-score subgroup than in the lower-score subgroup at a statistically significant level of 5\%. Moreover, a statistically significant trend of $10 \%$ was found that less subjects evaluated the agent with face image animation as chatty and pleasant in the ACAS-S2 higher-score subgroup than in the lower-score subgroup.

Thirdly, to investigate the effects of the subjects' anxiety toward social influences of computer technologies to their impression evaluation of the agents, the subject group was divided into two subgroups based on the median value of ACAS-S3 scores. As shown in table 3, the $t$-test for the ACAS-S3 scores revealed a statistically significant difference between these subgroups on anxiety toward social influences of computer technologies. Then, for each adjective, tests of independence were conducted for the numbers
Table 5. The Numbers of Subjects Who Selected the Agent with Face Image Animation for Each Impressive Adjective in ACAS-S2 Higher-Score and Lower Score Subgroups, and Results of Tests of Independence

\begin{tabular}{lccr}
\hline \hline & $\begin{array}{c}\text { Higher } \\
(N=8)\end{array}$ & $\begin{array}{c}\text { Lower } \\
(N=8)\end{array}$ & $p$ \\
\hline Pretty & 5 & 7 & .569 \\
Chatty & 4 & 8 & .077 \\
Hateful & 5 & 3 & .619 \\
Foolish & 7 & 2 & .041 \\
Clever & 3 & 4 & 1.000 \\
Dark & 3 & 0 & .200 \\
Familiar & 4 & 7 & .282 \\
Empty & 3 & 0 & .200 \\
Unpleasant & 7 & 3 & .119 \\
Considerate & 4 & 7 & .282 \\
Unfamiliar & 6 & 1 & .041 \\
Pleasant & 4 & 8 & .077 \\
Terrible & 5 & 2 & .315 \\
Full & 3 & 6 & .315 \\
Formal & 3 & 0 & .200 \\
Selfish & 7 & 5 & .569 \\
Mild & 3 & 6 & .315 \\
Light & 5 & 7 & .569 \\
\hline \hline
\end{tabular}

of subjects who selected the agent with face image animation in the same way.

As shown in table 6, a statistically significant trend of $10 \%$ was found that less subjects evaluated the agent with face image animation as pleasant and light in the ACAS-S3 higher-score subgroup than in the lower-score subgroup.

Fourthly, the correlation between the degrees of the subjects' positive impression for the agent with face image animation and computer anxiety was analyzed as follows. Each subject's score of positive impression for the agent with face image animation $P E V$ was defined as the sum of the numbers of positive adjectives for which the agent with face image animation was selected and those of negative adjectives for which the agent without face images was selected (Max: 18, Min: 0). As shown in table 7, Pearson's correlation coefficient $r$ between $P E V$ and the ACAS total scores revealed a moderate level of negative correlation between them, although it was a statistically significant trend of $10 \%$.

Finally, comparison of impression evaluation for the agents was conducted to verify the total effect of face image animation as follows. There was no statistically significant difference on the numbers of subjects who selected the agent with face image animation between the higher- 
Table 6. The Numbers of Subjects Who Selected the Agent with Face Image Animation for Each Impressive Adjective in ACAS-S3 Higher-Score and Lower Score Subgroups, and Results of Tests of Independence

\begin{tabular}{lccr}
\hline \hline & $\begin{array}{c}\text { Higher } \\
(N=8)\end{array}$ & $\begin{array}{c}\text { Lower } \\
(N=8)\end{array}$ & $p$ \\
\hline Pretty & 6 & 6 & 1.000 \\
Chatty & 5 & 7 & .569 \\
Hateful & 4 & 4 & 1.000 \\
Foolish & 5 & 4 & 1.000 \\
Clever & 2 & 5 & .315 \\
Dark & 2 & 1 & 1.000 \\
Familiar & 5 & 6 & 1.000 \\
Empty & 2 & 1 & 1.000 \\
Unpleasant & 5 & 5 & 1.000 \\
Considerate & 4 & 7 & .282 \\
Unfamiliar & 4 & 3 & 1.000 \\
Pleasant & 4 & 8 & .077 \\
Terrible & 3 & 4 & 1.000 \\
Full & 5 & 4 & 1.000 \\
Formal & 3 & 0 & .200 \\
Selfish & 6 & 6 & 1.000 \\
Mild & 4 & 5 & 1.000 \\
Light & 4 & 8 & .077 \\
\hline \hline
\end{tabular}

socre and lower-score subgroups of all the ACAS subscales in eleven adjectives ("Pretty," "Hateful,", and so on). On these adjectives, binomial tests with rate $50 \%$ for the complete samples were conducted to verify a bias of the rates in which the agent with face image animation was selected. The selection rates were lower than $50 \%$ at a statistically significant level of 5\% for "Dark," "Empty," and "Formal." Furthermore, interval estimation found that the selection rates of the agent with face image animation for these adjectives were within $[.040, .456]$ with confidence of $95 \%$.

\section{Discussion}

The results of the experiment show the following facts.

In the pseudo double-bind situation, the agent with face image animation gave the subjects less negative impression represented as dark, empty, and formal, than the agent without face images. In more details, however, impression of the agents changed dependent on the subjects' degrees of computer anxiety.

In comparison with the subjects with lower anxiety toward operation of computers, those with higher anxiety tended to evaluate that the agent without face images were more clever than the agent with face images. In compari-
Table 7. Means and Standard Deviations of Scores of Positive Impression for the Agent with Face Image Animation and ACAS Total Scores, and Pearson's Correlation Coefficient $r(N=16)$

\begin{tabular}{lcc}
\hline \hline & Mean & SD \\
\hline ACAS & 48.4 & 9.2 \\
PEV & 11.1 & 5.4 \\
& $r=-.488$ & $(p=.055)$ \\
\hline \hline
\end{tabular}

son with the subjects with lower anxiety toward approach to computers, those with higher anxiety tended to evaluate that the agent with face image animation were more foolish and unfamiliar than the agent without face images. Furthermore, there was a moderate level of negative correlation between the subjects' positive impression evaluation of the agent with face images and anxiety toward computers.

The above facts lead to the following implications.

In double-bind situations of human-agent interaction, users may have more positive impression for anthropomorphic agents like face images. However, users' personal traits such as computer anxiety may influence the effect. On people with higher anxiety toward computers, for example, the elderly people who have been inexperienced in computers, we may not be able to expect the effect of positive impression by anthropomorphication of agents, in comparison with younger people familiar with computers. In other words, the degree of anthropomorphizing agents should be modified dependent on users' personal traits in double-bind situations.

On the other hand, computer anxiety is a kind of state anxiety, that is, it is transiently evoked in specific situations that changes depending on the situation and time, and can be reduced by appropriate educational programs [8]. This fact implies that reduction of users' computer anxiety through appropriate educational methods may improve the effect of anthropomorphic agents. Moreover, Rickenberg and Reeves [18] found that monitoring behaviors of animated characters influenced users' anxiety and task performance. It implies the possibility of reducing users' computer anxiety by agent design itself. Thus, automatic recognition of users' computer anxiety by agents and modification mechanisms of their characteristics to reduce anxiety may contribute to application of agent technologies in real fields.

In case of mental therapeutic agents, clients' computer anxiety is a factor that double-bind situations consist of. If clients' computer anxiety is reduced by therapeutic agents themselves, doubled-bind situations will vanished and anthropomorphic agents may contribute to therapeutic effects, 
for example, by behavior strategy $[4,18]$ and affective expression [3]. If it is hard to implement agents' mechanisms of reducing users' computer anxiety, however, we should be careful for the usage of therapeutic agents.

\section{Limitation:}

Since the subjects in the experiment were all Japanese students and the sample size was small, whether the findings can be applied to people in other groups, such as different age groups or cultures is inconclusive. Moreover, we need to take into account other personal traits of users (e.g., locus of control [18]) and characteristics of agents such as behavior strategy and affective expression related to mental therapeutic aims $[2,4]$. These problems must be tackled in future experiments by extending the design, for example, by using between-subjects design based on large samples to clarify the interaction effect in more details.

Furthermore, we have not found any hypothesis on why users with high computer anxiety tend to avoid anthropomorphic agents in double-bind situations. In addition, the pseudo double-bind situation in the experiment is different from double-bind situations in human-agent interaction under mental therapeutic contexts, in the sense that in the former computer anxiety is independent on the doublebind situation, although in the latter it is one of factors that double-bind situations consist of. To solve these problems, we need to investigate psychological theories in more details to find the hypothesis and improve the experimental design.

\section{Acknowledgment}

The research was supported by the Japan Society for the Promotion of Science, Grants-in-Aid for Scientific Research No. 18500207, and by the "High-Tech Research Center" Project for Private Universities with a matching fund subsidy from MEXT (Ministry of Education, Culture, Sports, Science and Technology), 2002-2006.

\section{References}

[1] G. Bateson. Steps to an Ecology of Mind. Harper \& Row, 1972. (Japanese translation: Y. Sato. Shisaku-Sha, 1990.).

[2] T. W. Bickmore and R. W. Picard. Establishing and maintaining long-term human-computer interaction. ACM Transactions on Computer-Human Interaction, 12:293327, 2005.

[3] K. Blocher and R. W. Picard. Affective social quest: Emotion recognition therapy for autistic children. In K. Dautenhahn, A. H. Bond, L. Cañamero, and B. Edmonds, editors, Socially Intelligent Agents: Creating Relationships with Computers and Robots, chapter 16, pages 133-140. Kluwer Academic Publishers, 2002.

[4] S. Brave, C. Nass, and K. Hutchinson. Computers that care: investigating the effects of orientation of emotion exhibited by an embodied computer agent. International Journal of Human-Computer Studies, 62:161-178, 2005.

[5] L. Ciompi. Affektlogik. Klett-Cotta, 1982. (Japanese translation: M. Matsumoto et al. (1994). Gakuju Shoin).

[6] V. D. Foley. An Introduction to Family Therapy. Allyn \& Bacon, 1986. (Japanese translation: A. Fujinawa, et al. Sogensha, 1993.).

[7] M. Hase. A system theoretical approach to double bind. Japanese Sociological Review, 40:310-324, 1989. (in Japanese).

[8] K. Hirata. The concept of computer anxiety and measurement of it. Bulletin of Aichi University of Education, 39:203-212, February 1990. (in Japanese).

[9] A. R. Hochschild. The Managed Heart. University of California Press, 1983. (Japanese translation: J. Ishikawa and A. Murofushi. Sekaishishosha, 2000.).

[10] T. Kanda, H. Ishiguro, and T. Ishida. Psychological evaluation on interactions between people and robots. Journal of the Robotics Society of Japan, 19(3):362-381, 2001. (in Japanese).

[11] M. Koopmans. Schizophrenia and the Family: Double Bind Theory Revisited. Dynamical Psychology, 1997. http://goertzel.org/dynapsyc/1997/Koopmans.html (electric journal).

[12] S. C. Marsella. Pedagogical soap: Socially intelligent agents for interactive dramm. In K. Dautenhahn, A. H. Bond, L. Cañamero, and B. Edmonds, editors, Socially Intelligent Agents: Creating Relationships with Computers and Robots, chapter 17, pages 141-148. Kluwer Academic Publishers, 2002.

[13] T. Nomura. Double bind situations in man-machine interaction under contexts of mental therapy. In T. Rist, et. al., editor, Intelligent Virtual Agents: 4th International Workshop, IVA 2003, volume 2792 of LNAI, pages 67-71. Springer, 2003.

[14] K. Ohnishi and T. Nomura. Verification of mental influence in man-machine interaction based on double-bind theory. In Proc. 34th Annual Conference of International Simulation and Gaming Association (ISAGA), pages 315-323, 2003.

[15] K. Ohnishi and T. Nomura. Experimental verification of mental influence by double-bind theoretic software agents,. In Proc. Joint 2nd International Conference on Soft Computing and Intelligent Systems and 5th International Symposium on Advanced Intelligent Systems (SCIS \& ISIS 2004), 2004. (CD-ROM Proceedings).

[16] A. C. Raub. Correlates of computer anxiety in college students. PhD thesis, University of Pennsylvania, 1981.

[17] B. Reeves and C. Nass. Media Equation. Cambridge Press, 1996. (Japanese translation: H. Hosoma. Shoeisha, 2001.).

[18] R. Rickenberg and B. Reeves. The effect of animated characters on anxiety, task performance, and evaluations of user interfaces. In Proc. SIGCHI Conference on Human Factors in Computing Systems, pages 49-56, 2000.

[19] G. Ritzer. The McDonaldozation of Society. Pine Forge Press, 1996. (Japanese Edition: K. Masaoka (1999). Waseda University Press).

[20] M. Yamada. Social control by emotions. In M. Okahara, M. Yamada, H. Yasukawa, and J. Ishikawa, editors, The Sociology of Emotions, pages 69-90. Sekai-Shiso-Sha, Kyoto, Japan, 1997. (in Japanese). 